\title{
Compatibilização de Blendas de Poliamida 6/ABS usando os Copolímeros Acrílicos Reativos MMA-GMA e MMA-MA. Parte 2: Comportamento Termomecânico e Morfológico das Blendas
}

\author{
Edcleide M. Araújo \\ Departamento de Engenharia de Materiais, UFCG \\ Elias Hage Jr. \\ Departamento de Engenharia de Materiais, UFSCar
}

\author{
Antônio J. F. Carvalho \\ Instituto de Química de São Carlos, USP
}

\begin{abstract}
Resumo: Blendas poliméricas de poliamida 6 (PA6) com acrilonitrila-butadieno-estireno (ABS) foram preparadas em extrusora de rosca dupla, utilizando-se os copolímeros metacrilato de metila - metacrilato de glicidila (MMA-GMA) e metacrilato de metila-anidrido maléico (MMA-MA) como agentes de compatibilização. O ABS, por si só, não foi capaz de tenacificar a PA6, apresentando uma morfologia de fases com grandes e pequenos aglomerados na matriz PA6. A introdução do copolímero MMA-GMA, como compatibilizante do sistema, não melhorou significativamente as propriedades de impacto da blenda PA6/ ABS. As fotomicrografias obtidas por microscopia eletrônica de transmissão (MET) indicaram uma morfologia com duas populações distintas de ABS: aglomerados e pequenas partículas dispersas, resultando em uma distribuição não-uniforme de domínios de ABS. A blenda compatibilizada com MMA-MA foi supertenaz ( $>800 \mathrm{~J} / \mathrm{m})$ na temperatura ambiente e em baixas temperaturas $\left(\sim-10^{\circ} \mathrm{C}\right)$, com baixas concentrações de compatibilizante e baixos teores de MA no copolímero. As blendas PA6/ ABS compatibilizadas com MMA-MA apresentaram uma morfologia de partículas bem dispersas e adequadamente distribuídas na matriz, evidenciando a presença efetiva do copolímero como agente de compatibilização reativo deste sistema.
\end{abstract}

Palavras-chave: Morfologia, tenacificação, poliamida 6, ABS.

\section{Compatibilization of Polyamide 6/ABS Blends Using MMA-GMA and MMA-MA Reactive Acrylic Copolymers. Part 2. Thermal-Mechanical and Morphological Behavior of Blends}

\begin{abstract}
Blends of Polyamide 6 (PA6) with acrylonitrile-butadiene-styrene (ABS) were prepared in a corotating twin-screw extruder, using the poly(methyl methacrylate-co-glycidyl methacrylate) (MMA-GMA) and poly(methyl methacrylate-comaleic anhydride) (MMA-MA) copolymers as compatibilizing agents. The ABS by itself was not capable to toughen PA6 and showed a phase morphology with large and small agglomerates in the PA6 matrix. The introduction of MMA-GMA copolymer as a compatibilizing agent in the system did not significantly improve the impact properties of PA6/ABS blend. Transmission electron microscope (TEM) photomicrographs indicated a morphology with two distinct populations of ABS: agglomerates and small dispersed particles resulting in a non-uniform distribution of ABS domains. The compatibilized blend with MMA-MA was super-tough $(>800 \mathrm{~J} / \mathrm{m})$ at room temperature and low temperature $\left(\sim-10^{\circ} \mathrm{C}\right)$ with small amounts of MA in the copolymer and small amounts of compatibilizer in the blend. The PA6/ABS compatibilized blends with MMAMA showed a morphology of well dispersed and distributed rubber particles in PA6 matrix, thus demonstrating the effective presence of the copolymer as a compatibilizing reactive agent of this system.
\end{abstract}

Keywords: Morphology, toughness, polyamide, ABS.

\section{Introdução}

A poliamida 6 (PA6) é um polímero semicristalino que tem boa resistência química em relação aos meios orgânicos, baixa viscosidade no estado fundido e é muito atra- tivo para aplicações de engenharia. No entanto, a sua sensibilidade à propagação de trinca, alta absorção de umidade, estabilidade dimensional pobre, temperaturas de deflexão térmica relativamente baixas e fragilidade em temperaturas sub-ambientes tornam seu uso restrito a algumas

Autor para correspondência: Edcleide M. Araújo, DEMa, UFCG, Caixa Postal 10034, CEP:58109-970, Campina Grande, PB. E-mail: edcleide@dema.ufcg.edu.br 
aplicações. Essas deficiências podem ser melhoradas pela mistura com outros plásticos como poli(óxido de fenileno) (PPO), poli(acrilonitrila-butadieno-estireno) (ABS), poliolefinas, entre outros ${ }^{[1,2]}$. O ABS é um termoplástico, de menor viscosidade que um elastômero e muito utilizado devido à sua excelente tenacidade e baixo custo. A incorporação do copolímero $\mathrm{ABS}$ pode melhorar a resistência ao impacto sob entalhe da PA6, pois sua fase elastomérica, à base de polibutadieno (PB), apresenta um enorme potencial para aumentar a tenacidade da mistura final, bem como, diminuir a elevada absorção de umidade da $\mathrm{PA}^{[3-11]}$. Entretanto, como essa mistura é imiscível e incompatível, faz-se necessário o desenvolvimento de compatibilizantes capazes de reagir com os grupos terminais da PA6 e que sejam miscíveis com a fase estireno-acrilonitrila (SAN) do copolímero ABS, na interface PA6-SAN ${ }^{[2,5,12,13]}$. Recentemente, foi mostrada a influência de dois compatibilizantes, os copolímeros metacrilato de metila-metacrilato de glicidila (MMA-GMA) e metacrilato de metila-anidrido maléico (MMA-MA), nas propriedades reológicas e mecânicas da blenda PA6/ABS ${ }^{[11]}$. A escolha desses compatibilizantes se deve ao fato de que o poli(metacrilato de metila) (PMMA) é miscível com a fase SAN do ABS, conforme a literatura ${ }^{[14,15]}$; o grupo epóxi presente no GMA é capaz de reagir com as extremidades de cadeia da PA6 ${ }^{[16-23]}$; o anidrido maléico (MA) é capaz de reagir com os grupos amina da PA6 ${ }^{[24-28]}$. A blenda PA6/ABS/ MMA-MA apresentou excelente desempenho sob impacto, atingindo valores acima de $800 \mathrm{~J} / \mathrm{m}$, ou seja, supertenaz e com baixos conteúdos de MA (3\%), evidenciando a presença efetiva do copolímero, à base de MA, como agente de compatibilização reativo deste sistema. O objetivo deste trabalho foi mostrar a influência dos compatibilizantes MMAGMA e MMA-MA no comportamento termomecânico e morfológico das blendas PA6/ABS.

\section{Experimental}

A Tabela 1 apresenta os materiais usados neste trabalho. A PA6 (Ultramid B3), foi fornecida pela Basf, na forma de grânulos. O ABS foi fornecido pela Nitriflex S.A., na forma de pó. Os compatibilizantes reativos foram sintetizados em laboratório. A síntese e a caracterização dos copolímeros estão descritas detalhadamente na literatura ${ }^{[1,11]}$. Os procedimentos para a preparação das blendas, avaliação da reatividade e caracterização mecânica foram descritos recentemente ${ }^{[11]}$.

A temperatura de deflexão térmica (HDT) foi obtida através de corpos de prova de HDT, conforme a norma ASTM D648, em um equipamento Ceast, modelo HDT 6 VICAT P/ N 6921.000, com uma tensão de $1800 \mathrm{kPa}$, taxa de aquecimento de $120^{\circ} \mathrm{C} / \mathrm{h}$ (método A), onde o meio de imersão foi um óleo de silicone. As amostras foram acondicionadas a $23 \pm 2{ }^{\circ} \mathrm{C}$ e a umidade relativa do ar a $55 \pm 10 \%$, por pelo menos 48 horas antes da realização do ensaio. Uma série de seis amostras foi ensaiada e a temperatura de deflexão média, com seu respectivo desvio-padrão, reportados.

A análise térmica dinâmico-mecânica foi realizada em
Tabela 1. Materiais usados neste trabalho

\begin{tabular}{|c|c|c|c|}
\hline Polímero & Composição & $\begin{array}{c}\text { Peso Molecular } \\
\text { (g/mol) }\end{array}$ & $\begin{array}{c}\text { Torque Haake }^{\mathrm{d}} \\
\text { (Nm) }\end{array}$ \\
\hline Poliamida 6 & $\begin{array}{l}\mathrm{GTA}=43,2 \mu \mathrm{eqg}^{-1} \\
\mathrm{GTC}=51,5 \mu \mathrm{eqg}^{-1}\end{array}$ & $\mathrm{Mn}=21000^{\mathrm{a}}$ & 1,07 \\
\hline $\mathrm{ABS}$ & $\begin{array}{l}50 \% \text { PB e } 25 \% \text { AN } \\
\text { em SAN }\end{array}$ & $\begin{array}{c}\mathrm{Mn}=40000^{\mathrm{b}} \\
\mathrm{Mw}=110000^{\mathrm{b}}\end{array}$ & 10,4 \\
\hline MMA-GMA & $10 \%$ GMA & $\begin{array}{l}\mathrm{Mn}=21600^{\mathrm{c}} \\
\mathrm{Mw}=91300^{\mathrm{c}}\end{array}$ & 0,1 \\
\hline \multirow{4}{*}{ MMA-MA } & $3 \% \mathrm{MA}$ & $\begin{array}{l}\mathrm{Mn}=20900^{\circ} \\
\mathrm{Mw}=40400^{\mathrm{c}}\end{array}$ & 0,01 \\
\hline & $5 \% \mathrm{MA}$ & $\begin{array}{l}\mathrm{Mn}=15800^{\mathrm{c}} \\
\mathrm{Mw}=37900^{\mathrm{c}}\end{array}$ & 0,01 \\
\hline & $10 \% \mathrm{MA}$ & $\begin{array}{l}\mathrm{Mn}=13100^{\mathrm{c}} \\
\mathrm{Mw}=26200^{\mathrm{c}}\end{array}$ & 0,01 \\
\hline & $20 \%$ MA & $\begin{array}{l}\mathrm{Mn}=10000^{\mathrm{c}} \\
\mathrm{Mw}=16600^{\mathrm{c}}\end{array}$ & 0,01 \\
\hline
\end{tabular}

${ }^{a}$ Medido por análise química dos grupos terminais de cadeia

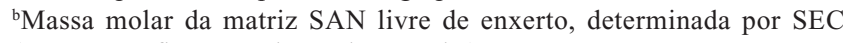
(Cromatografia por exclusão de tamanho)

'Determinado por SEC

${ }^{\mathrm{d}}$ Torque obtido após 10 min. de corrida.

um equipamento de DMTA da Polymer Laboratories, modelo Mk II. Os corpos de prova para este ensaio foram os utilizados para o teste de impacto Izod sem entalhe, com largura de $12,7 \mathrm{~mm}$, espessura de $3,2 \mathrm{~mm}$. O equipamento foi operado no modo flexão em três pontos, a uma freqüência de $1 \mathrm{~Hz}$, taxa de aquecimento de $3{ }^{\circ} \mathrm{C} / \mathrm{min}$, intervalo de temperatura de -100 a $150{ }^{\circ} \mathrm{C}$ e deformação de $64 \mu \mathrm{m}$. Os gráficos do módulo de armazenamento (E'), o módulo de perda (E”) e a tangente de perda ( $\tan \delta$ ) em função da temperatura foram registrados.

A morfologia das blendas foi analisada pela observação de imagens obtidas em um microscópio eletrônico de transmissão (MET), marca Philips CM 120, operando a uma voltagem de aceleração de $120 \mathrm{kV}$. Os corpos de prova do tipo Izod foram submetidos ao "trimer", a partir de barras Izod cortadas perpendiculares à direção do fluxo de injeção, na região do entalhe. Em seguida, foram criogenicamente microtomadas em seções ultrafinas, $20 \mathrm{~nm}$ de espessura, com facas de diamante, por um micrótomo tipo Riechert-Jung Ultracut $\mathrm{E}$, em torno de $-50^{\circ} \mathrm{C}$. Duas técnicas de tingimento foram utilizadas: as seções foram expostas a $2 \%$ de solução aquosa de ácido fosfotúngstico (PTA) por 40 minutos, para tingir a fase da poliamida e algumas amostras foram expostas ao vapor de tetróxido de ósmio $\left(\mathrm{OsO}_{4}\right)$ por 15 horas, para tingir a fase borrachosa do ABS. As imagens observadas no microscópio foram obtidas por meio de filmes fotográficos.

\section{Resultados e Discussão}

Os resultados de propriedades mecânicas sob tração e sob impacto para todas as blendas foram apresentados anteriormente ${ }^{[11]}$. Embora o ABS possua elevada resistência ao impacto, devido à existência de uma fase borrachosa em sua microestrutura, a incorporação deste na PA6 não aumentou 


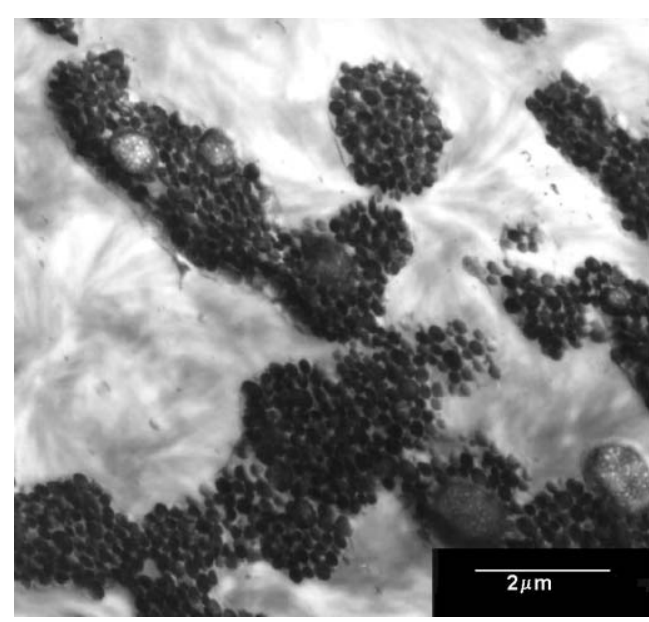

(a)

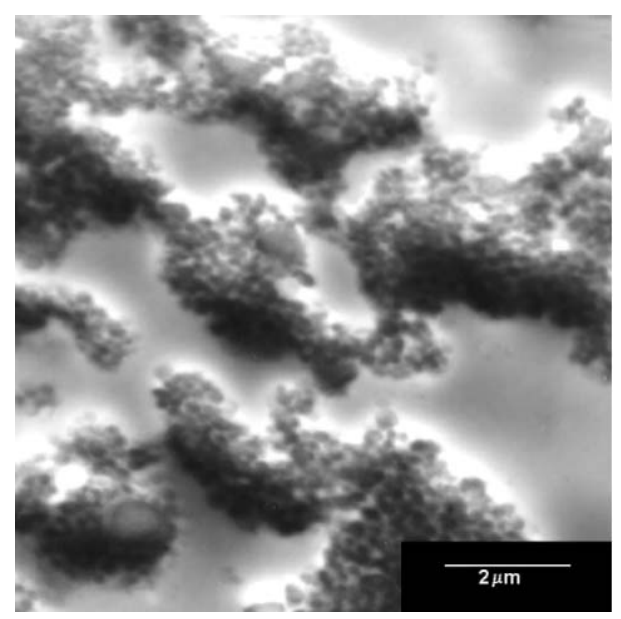

(b)

Figura 1. Fotomicrografias de MET para as blendas binárias PA6/ABS: (a) 70/30 \%; (b) 50/50 \%. A fase borrachosa insaturada do ABS foi tingida com $\mathrm{OsO}_{4}$ e aparece como fase escura (aumento de $8000 \mathrm{x}$ ).

suficientemente sua resistência ao impacto. $\mathrm{O}$ aspecto de fratura pode ser considerado frágil em função dos valores obtidos. Observou-se, também, que a incorporação do copolímero MMA-GMA proporcionou um aumento significativo na resistência ao impacto destas blendas, em relação à nãocompatibilizada. Muito embora o valor desta propriedade não possa ser comparado aos valores obtidos por Kudva et al. ${ }^{[17]}$, essa blenda pode ser considerada tenacificada $(\sim 200 \mathrm{~J} / \mathrm{m})$ na temperatura ambiente, comparada a outros plásticos vítreos tenacificados como o poliestireno de alto impacto (HIPS), que tem resistência ao impacto na ordem de $100 \mathrm{~J} / \mathrm{m}$.

Entretanto, os resultados de resistência ao impacto Izod para amostras entalhadas de PA6/ABS com o copolímero MMA-MA, mostraram um aumento muito significativo nesta propriedade. Os valores de resistência ao impacto alcançados em torno de $800 \mathrm{~J} / \mathrm{m}$ são superiores ao do próprio $\mathrm{ABS}$ sozinho, indicando um estado de sinergismo em relação a essa propriedade. A presença do compatibilizante torna a blenda supertenaz, comparável aos resultados obtidos por Majumdar et al. ${ }^{[9]}$, o que pode ser também observado na morfologia, já que esta é responsável pelo comportamento mecânico das blendas.

\section{Análise Morfológica}

A Figura 1 apresenta as fotomicrografias das blendas PA6/ ABS contendo composições variadas: $70 / 30$ e 50/50 \%. As amostras foram tingidas com $\mathrm{OsO}_{4}$. Em (a), blenda PA6/ABS (70/30 \%), observa-se um conteúdo um pouco menor de fase dispersa em relação a (b), blenda PA6/ABS (50/50 \%), que, além do maior conteúdo, apresenta maiores aglomerados de borracha. Observa-se, também, que a blenda contém domínios de ABS alongados com algum grau de co-continuidade. As propriedades mecânicas dessas blendas estão correlacionadas com este tipo de morfologia. A blenda da Figura 1 (a) apresenta resistência ao impacto na temperatura ambiente menor que a blenda com maior conteúdo de borracha, Figura 1 (b). Não há uma temperatura de transição dúctil-frágil para tais blendas, pois em toda a faixa de temperatura estudada, elas permanecem frágeis ${ }^{[11]}$. Em geral, a adição de um compatibilizante tem a finalidade de diminuir a tensão interfacial entre matriz e fase dispersa. As blendas sem compatibilizantes, na maioria das vezes, apresentam morfologia instável e, em certas situações de processamento, o nível de dispersão pode ser inadequado quando se quer boas propriedades. A introdução de compatibilizantes pode estabilizar a morfologia da blenda contra a coalescência, quando esta for exposta a condições de fusão em baixos cisalhamentos, por períodos de tempos prolongados ${ }^{[19]}$. Desta forma, são avaliados os efeitos de dois tipos de compatibilizantes na morfologia de blendas de PA6/ABS: o copolímero MMA-GMA e o copolímero MMA-MA. A Figura 2 mostra as fotomicrografias das blendas ternárias PA6/ ABS/MMA-GMA com 10\% GMA no copolímero. Na Figura 2 (a) $(66,5 / 28,5 / 5 \%$ ), a presença do copolímero MMAGMA parece interromper a co-continuidade dos domínios de ABS [Figura 1 (a)], ou seja, é como se o compatibilizante reestruturasse os domínios de $\mathrm{ABS}$ em duas populações: aglomerados e domínios pequenos. Os domínios de ABS parecem se tornar mais eficientemente dispersos. Esse melhoramento na dispersão é acompanhado por um pequeno aumento na resistência ao impacto na temperatura ambiente dessas blendas compatibilizadas em relação à nãocompatibilizada $^{[11]}$. Observa-se, também, para todas as seqüências, que não há diferença significativa da morfologia de uma seqüência em relação à outra. A Figura 2 (b) apresenta a fotomicrografia para a blenda ternária PA6/ABS/MMAGMA (47,5/47,5/5\%). Comparando-se a morfologia desta seqüência com a morfologia apresentada na Figura 1 (b) para a blenda binária, observa-se que os grandes aglomerados de ABS na blenda binária parecem ter sido interrompidos, apresentando-se mais dispersos; entretanto, há tendência à co-continuidade. Essa seqüência apresenta resistência ao impacto na temperatura ambiente um pouco melhor do que as anteriores (acima de $200 \mathrm{~J} / \mathrm{m}$ ) e do que a blenda sem 


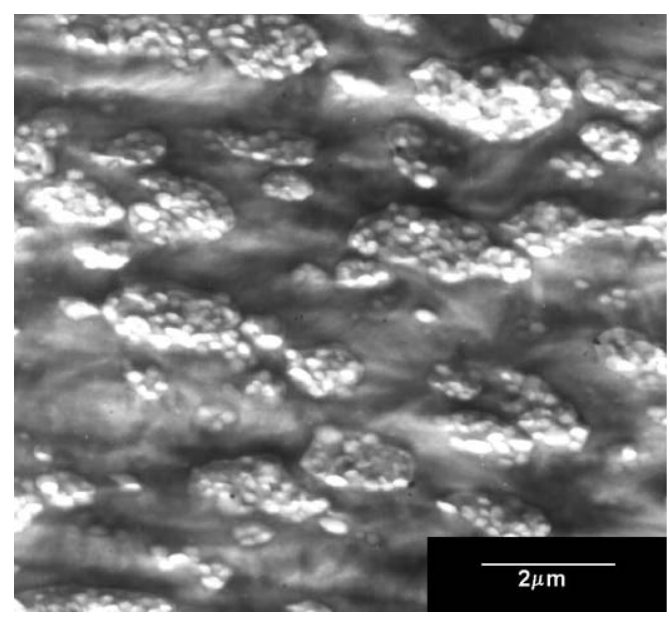

(a)

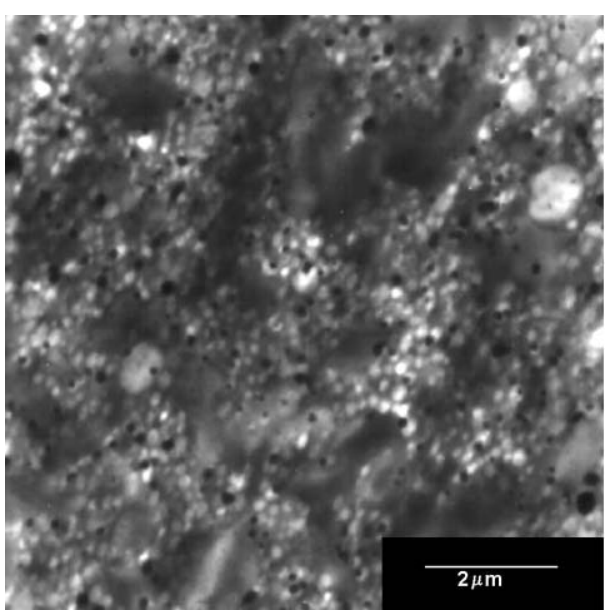

(b)

Figura 2 - Fotomicrografias de MET para as blendas ternárias (a) PA6/ABS/MMA-GMA (66,5/28,5/5 \%) - seqüência 3 e (b) PA6/ABS/MMA-GMA $(47,5 / 47,5 / 5 \%)$ - seqüência 5. A fase de PA6 foi tingida com PTA e aparece como uma região escura (aumento de 8000 x).

compatibilizante. Esse resultado pode estar relacionado ao fato de se ter um maior conteúdo de borracha nestas blendas. A dificuldade do copolímero MMA-GMA em produzir domínios bem dispersos de ABS em PA6 já foi mencionada na literatura ${ }^{[17]}$, e se deve, provavelmente, à difuncionalidade da PA6 em relação ao grupo epóxi do GMA. Tanto a amina quanto o ácido, ambos grupos finais da PA6, podem reagir com os anéis epóxi, conduzindo a efeitos do tipo reticulação, que dificultam a boa dispersão dos domínios de ABS. Isso é o que acontece nas blendas de poliamida 6,6 , em que algumas cadeias têm dois grupos finais amina, com ABS contendo um compatibilizante do tipo anidrido. Por outro lado, contaminantes resultantes da polimerização por emulsão do ABS, do tipo íons metálicos e emulsificante, podem influenciar as reações com epóxis ${ }^{[19]}$. Portanto, o copolímero MMAGMA não demonstrou ser um compatibilizante eficiente para as blendas de PA6/ABS estudadas neste trabalho. A Figura 3 mostra as blendas ternárias de PA6/ABS/MMA-MA

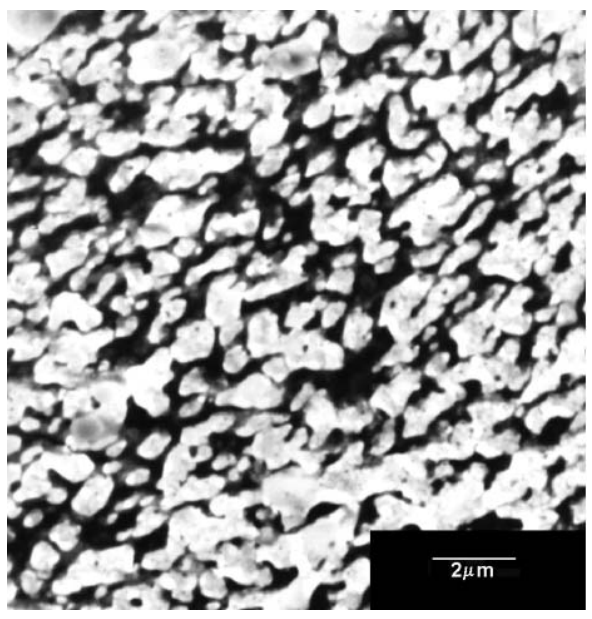

(a)
$(47,5 / 47,5 / 5 \%)$ com quantidades variadas de anidrido maléico no copolímero (a) $5 \%$ e (b) $10 \%$. Todas as amostras foram tingidas com PTA. Na Figura 3 (a), observa-se uma região escura bem definida, que é a PA6, e glóbulos grandes e pequenos de ABS. Há tendência à co-continuidade da fase $\mathrm{ABS}$ e não dá para argumentar qual é a fase contínua e a fase dispersa. Na Figura 3 (b), observa-se, claramente, uma fase contínua de PA6 (escura) e domínios bem dispersos e pequenos da fase ABS, com algum grau de cocontinuidade. Todas estas blendas foram supertenazes (acima de $800 \mathrm{~J} / \mathrm{m}$ ) na temperatura ambiente e permaneceram assim em temperaturas sub-ambiente. A temperatura de transição dúctil-frágil para as blendas compatibilizadas com $3,5,10$ e $20 \%$ MA no copolímero foi $-10,-10,-2,5$ e $28^{\circ} \mathrm{C}$, respectivamente ${ }^{[1,11]}$. As blendas com 3 e $5 \% \mathrm{MA}$ no copolímero apresentaram comportamento mecânico muito semelhante. A blenda com $20 \% \mathrm{MA}$ tornou-se frágil mais rapidamente, ou seja, em temperatura mais alta do que as

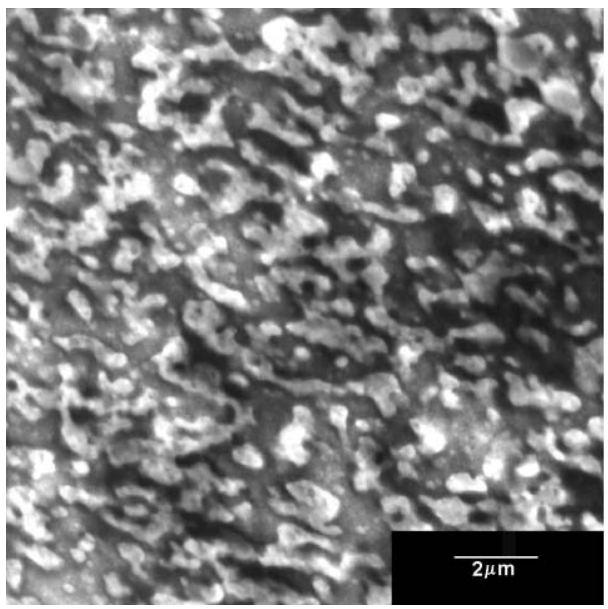

(b)

Figura 3 - Fotomicrografias de MET para as blendas ternárias PA6/ABS/MMA-MA (47,5/47,5/5 \%) contendo quantidades variadas de anidrido maléico no copolímero: (a) $5 \%$ e (b) $10 \%$. A fase de PA6 foi tingida com PTA e aparece como uma região escura (aumento de 5000x). 


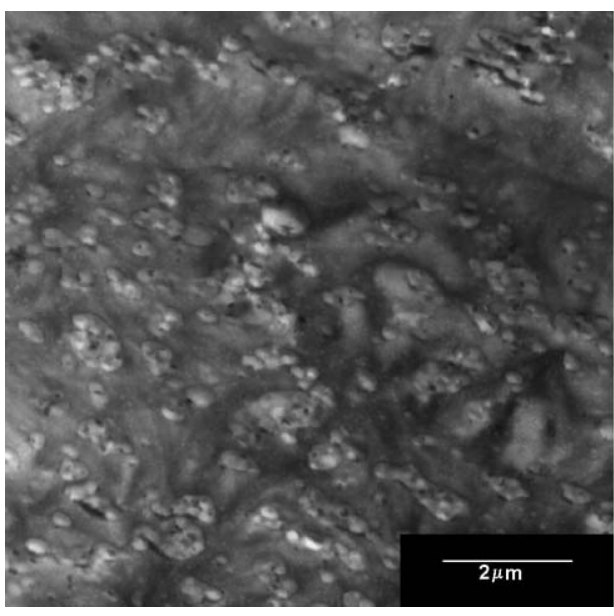

(a)

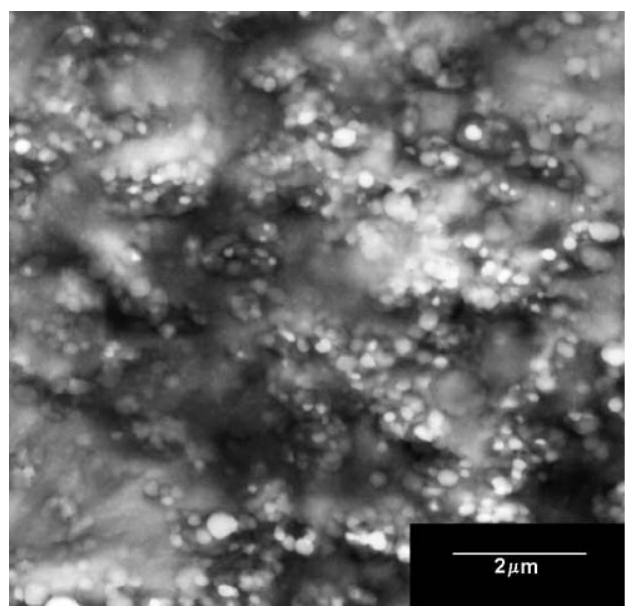

(b)

Figura 4. Fotomicrografias de MET para as blendas ternárias PA6/ABS/MMA-MA contendo $5 \%$ de anidrido maléico no copolímero: (a) 66,5/28,5/5 \% e (b) $68,25 / 29,25 / 2,5 \%$. A fase de PA6 foi tingida com PTA e aparece como uma região escura (aumento de 8000 x).

demais. Talvez, por esse copolímero apresentar uma massa molar menor do que os outros copolímeros e, em princípio, massa molar mais alta, proporciona melhores propriedades para qualquer sistema polimérico ${ }^{[29]}$. Uma outra hipótese seria observar a morfologia com o copolímero com $10 \%$ MA, Figura 3 (b). Ela apresenta um tamanho de partícula menor do que as demais, ou seja, provavelmente um tamanho inferior ao limite crítico para tenacificação, como mencionado na literatura ${ }^{[25,28]} \mathrm{e}$ argumenta-se que um aumento na quantidade de grupos funcionais reduz o tamanho da fase dispersa e melhora as propriedades de impacto, se as partículas forem originalmente grandes o suficiente para a tenacificação efetiva ${ }^{[27]}$. As partículas de borracha devem funcionar não apenas iniciando os mecanismos de tenacificação, que começam ao redor delas, mas também retardando a fratura. Portanto, o tamanho e a distribuição de partículas são de fundamental importância no desenvolvimento destes mecanismos ${ }^{[30]}$. Não apenas o tamanho das partículas, mas a distância entre estas influencia as propriedades, particularmente, a transição dúctil-frágil ${ }^{[31]}$. Lu et al. ${ }^{[21]}$ argumentaram que a distância crítica entre as partículas é uma propriedade do material da matriz, ou seja, a matriz é que deve ter a capacidade para escoar e isso independe do conteúdo de borracha e do tamanho dos domínios. Mesmo com baixos conteúdos de borracha, se esta se aglomerar, não haverá tenacificação. Entretanto, altos conteúdos e má dispersão e distribuição, também, favorecem a não-tenacificação. Outras variáveis também como condições de processamento devem favorecer a dispersão e distribuição das partículas de borracha, para que essas tenham uma distância ótima entre partículas. Outras composições foram estudadas utilizando-se o mesmo sistema de copolímero: a composição 66,5/28,5/5\% e 68,25/29,25/2,5\%. O objetivo era garantir que a matriz fosse a PA6 e, ao mesmo tempo, estudar o comportamento deste copolímero na blenda de PA6/ABS, com um conteúdo menor de ABS. A Figura 4 apresenta as fotomicrografias das blendas PA6/ABS/ MMA-MA com $5 \%$ MA no copolímero com (a) 66,5/28,5/5\% e (b) $68,25 / 29,25 / 2,5 \%$. Comparando-se essas morfologias com a da Figura 1 (a), observa-se que o compatibilizante utilizado diminuiu significativamente o tamanho dos domínios de $\mathrm{ABS}$ que se encontram uniformemente distribuídos e dispersos. Essa alteração na morfologia foi acompanhada por um aumento significativo na resistência ao impacto destas blendas, na temperatura ambiente. Embora elas tenham permanecido tenazes em temperatura inferior $\left(15^{\circ} \mathrm{C}\right)$, as temperaturas de transição dúctil-frágil foram maiores $\left(\sim 9,4^{\circ} \mathrm{C}\right)$ do que as anteriores com composição $50 / 50 \%$, provavelmente devido ao maior conteúdo de PA6 nestas blendas ${ }^{[1,11]}$. Na Figura 4 (b) os domínios de ABS parecem ser um pouco maiores do que os da anterior, Figura 4 (a), com tendência à coalescência. Mas, mesmo com baixo teor de copolímero, a estrutura aparece refinada em relação à blenda sem compatibilizante, Figura 1 (a). A blenda apresentou-se tenaz na temperatura ambiente e até $15^{\circ} \mathrm{C}$, com temperatura de transição dúctil-frágil na faixa de $11^{\circ} \mathrm{C}$, ou seja, comportamento semelhante ao da Figura 4 (a). Portanto, o conteúdo de grupos funcionais e a composição das blendas são fatores que podem determinar o tamanho e a distribuição da fase dispersa e, portanto, o processo de tenacificação. Por outro lado, a boa eficiência do copolímero MMA-MA pode estar relacionada ao fato de que esse é miscível com a fase SAN do ABS e é capaz de reagir apenas com os grupos terminais amina da PA6, ou seja, provavelmente o compatibilizante (MMA-MA) tem a capacidade de formar copolímeros enxertados na interface poliamida-SAN, durante o processamento, evitando assim a coalescência das partículas da fase dispersa.

\section{Análise Termo-mecânica}

A análise termo-mecânica pode ser utilizada para avaliar a compatibilidade dos sistemas poliméricos de forma semelhante às propriedades mecânicas ${ }^{[32]}$.

A Figura 5 mostra os dados obtidos para a temperatura de deflexão térmica da PA6 pura e das blendas poliméricas com composição $68,25 / 29,25 / 2,5 \%$, com 3 e $5 \%$ de anidrido 


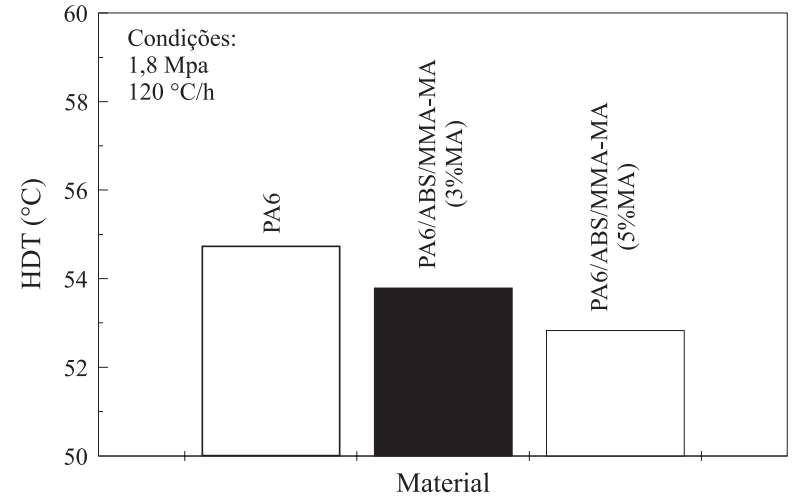

Figura 5. Temperatura de deflexão térmica (HDT) das blendas PA6/ABS/ $\operatorname{MMA}(68,25 / 29,25 / 2,5 \%)$ e da PA6 Pura. maléico no copolímero. Embora o teor de ABS seja em torno de $30 \%$ e $2,5 \%$ de compatibilizante tenha sido adicionado na blenda, o objetivo desta determinação foi comparar a tendência do comportamento da HDT para o componente puro e sua mistura. A HDT da PA6 pura ficou na faixa de $55^{\circ} \mathrm{C}$ e das blendas na faixa de 54 e $53{ }^{\circ} \mathrm{C}$ para aquelas com 3 e $5 \%$ de MA no copolímero, respectivamente. Portanto, não há diferença significativa nestes valores para esta propriedade. Jang et al. ${ }^{[4]}$, analisando o sistema PA6/ABS, com ABS modificado com anidrido maléico (ABS-MA), verificaram que a HDT da blenda com ABS-MA subiu quase que linearmente com o aumento da concentração de PA6, enquanto que a blenda com ABS não foi significativamente afetada pela adição de PA6, ou seja, as temperaturas de deflexão térmicas das blendas PA6/ABS-MA são bem mais altas do que aquelas

Tabela 2. Temperatura de transição vítrea e transições secundárias obtidas das curvas de $\tan \delta$ no ensaio de DMTA

\begin{tabular}{|c|c|c|c|c|c|}
\hline \multirow[b]{2}{*}{$\begin{array}{c}\text { Material } \\
\text { PA6/ABS/Cop.(\%) }\end{array}$} & \multicolumn{3}{|c|}{ ABS } & \multicolumn{2}{|c|}{ PA6 } \\
\hline & $\begin{array}{c}\operatorname{Tg}\left({ }^{\circ} \mathrm{C}\right) \\
\text { Fase rica em PB }\end{array}$ & $\begin{array}{c}\mathrm{T}_{1}\left({ }^{\circ} \mathrm{C}\right) \\
\text { Fase rica em PB }\end{array}$ & $\begin{array}{c}\mathrm{Tg}\left({ }^{\circ} \mathrm{C}\right) \\
\text { Fase rica em SAN }\end{array}$ & $\mathbf{T}_{2}\left({ }^{\circ} \mathbf{C}\right)$ & $\operatorname{Tg}\left({ }^{\circ} \mathrm{C}\right)$ \\
\hline $100 / 0 / 0$ & - & - & - & $-54,7$ & 57,9 \\
\hline 0/100/0 & $-65,9$ & $-40,6$ & 112,0 & - & - \\
\hline 70/30/0 & $-69,5$ & $-46,6$ & 110,5 & 102,1 & 55,9 \\
\hline Seqüência 1 & $-70,2$ & $-47,2$ & 102,8 & - & 41,2 \\
\hline Seqüência 2 & $-70,9$ & $-48,2$ & 103,5 & - & 39,5 \\
\hline Seqüência 3 & $-70,9$ & $-47,5$ & 102,0 & - & 37,2 \\
\hline Sequiência 4 & $-70,1$ & $-48,4$ & 108,7 & - & 44,3 \\
\hline $\begin{array}{l}66,5 / 28,5 / 5 \\
5 \% \mathrm{MA}\end{array}$ & $-69,6$ & $-45,9$ & 108,7 & 102,8 & 55,4 \\
\hline $\begin{array}{l}66,5 / 28,5 / 5 \\
3 \% \mathrm{MA}\end{array}$ & $-69,9$ & $-47,3$ & 110,1 & 104,3 & 56,7 \\
\hline $\begin{array}{l}68,25 / 29,25 / 2,5 \\
5 \% \mathrm{MA}\end{array}$ & $-68,5$ & $-45,8$ & 112,2 & 103,5 & 54,3 \\
\hline $\begin{array}{l}68,25 / 29,25 / 2,5 \\
3 \% \mathrm{MA}\end{array}$ & $-69,2$ & $-47,3$ & 111,5 & 105,0 & 52,3 \\
\hline $50 / 50 / 0$ & $-69,2$ & $-45,1$ & 110,8 & 103,6 & 56,0 \\
\hline Seqüência 5 & $-70,0$ & $-45,8$ & 112,2 & 107,1 & 45,7 \\
\hline Seqüência 6 & $-69,2$ & $-44,3$ & 111,5 & 107,8 & 44,2 \\
\hline $\begin{array}{l}47,5 / 47,5 / 5 \\
5 \% \mathrm{MA}\end{array}$ & $-68,5$ & $-45,1$ & 105,7 & - & 47,2 \\
\hline $\begin{array}{l}47,5 / 47,5 / 5 \\
3 \% \mathrm{MA}\end{array}$ & $-69,2$ & $-45,2$ & 107,3 & - & 52,3 \\
\hline
\end{tabular}

$\mathrm{T}_{1}$ : Temperatura de transição secundária referente à fase rica em PB do ABS

$\mathrm{T}_{2}$ : Temperatura de transição secundária referente à PA6, no seu estado puro, e referente à fase rica em SAN, em algumas blendas

Seqüência 1 - PA6/MMA-GMA + ABS $(66,5 / 5+28,5 \%)$

Seqüência 2 - ABS/MMA-GMA + PA6 $(28,5 / 5+66,5 \%)$

Seqüência 3 - PA6/ABS + MMA-GMA $(5+66,5 / 28,5 \%)$

Seqüência 4 - PA6/MMA-GMA + ABS/MMA-GMA (66,5/2,5 + 28,5/2,5\%)

Seqüência 5 - PA6/ABS/MMA-GMA (47,5/47,5/5\%)

Seqüência 6 - ABS/MMA-GMA + PA6 (47,5/5 + 47,5\%) 
para as blendas PA6/ABS, para todas as composições estudadas. Os valores observados para as blendas neste trabalho são muito interessantes do ponto de vista tecnológico, pois eles estão muito próximos aos da PA6, isto é, sem perdas da HDT.

A técnica de análise térmica dinâmico-mecânica, DMTA, foi utilizada para a determinação de transições, normalmente insignificantes para serem detectadas por técnicas convencionais estáticas como DSC. Como os materiais poliméricos são viscoelásticos, quando são solicitados por uma tensão senoidal, eles respondem também senoidalmente, porém esta deformação é atrasada em relação à solicitação. Esse comportamento pode ser acompanhado por DMTA, por meio da análise da movimentação molecular. O módulo de armazenamento ou E' é o módulo relativo à componente elástica; o módulo relativo à componente plástica, também chamado módulo de perda é o E". O amortecimento ou tangente de perda, $\tan \delta$, pode ser obtido pela relação entre os módulos de perda e armazenamento, ou seja, a razão adimensional entre as componentes viscosa e elástica, respectivamente ${ }^{[33]}$.

A Tabela 2 apresenta os resultados obtidos por DMTA para as blendas. As Figuras 6 e 7 mostram as curvas de E" e tan $\delta$, respectivamente, em função da temperatura para as blendas PA6/ABS/MMA-GMA (47,5/47,5/5\%). As curvas das blendas com o copolímero MMA-MA, apresentam comportamento semelhante às figuras citadas. Os picos em $\tan \delta$ definem a temperatura de transição dos componentes. Para a PA6 foram encontradas duas transições em $-54,7$ e $57,9^{\circ} \mathrm{C}$. A primeira pode estar relacionada à relaxação $\beta$ e é atribuída à mobilidade resultante da atração entre o $\mathrm{H}$ de uma cadeia molecular e os grupos $\mathrm{C}=\mathrm{O}$ da outra cadeia. A outra transição seria a própria $\mathrm{Tg}$, atribuída à relaxação $\alpha$, conforme literatura ${ }^{[34]}$. Para o ABS foram observadas três transições. A primeira, $-65,9^{\circ} \mathrm{C}$, é atribuída à $\mathrm{Tg}$ da fase rica em $\mathrm{PB}$; a segunda, $-40,6{ }^{\circ} \mathrm{C}$, pode ser atribuída a uma transição secundária e, a terceira, $112,0{ }^{\circ} \mathrm{C}$, seria a $\mathrm{Tg}$ da fase rica em SAN. Para as blendas, em geral, pode ser observado que as Tg's se situam em torno das Tg's dos materiais em seu estado puro, ou seja, dois picos de $\tan \delta$ indicam que o sistema é imiscível para todas as composições estudadas ${ }^{[35]}$. Não foram verificadas alterações significativas nas temperaturas $\mathrm{Tg}$ e $\mathrm{T}_{1}$ da fase rica em PB no ABS, para todas as blendas. As diferenças nos valores observados podem estar

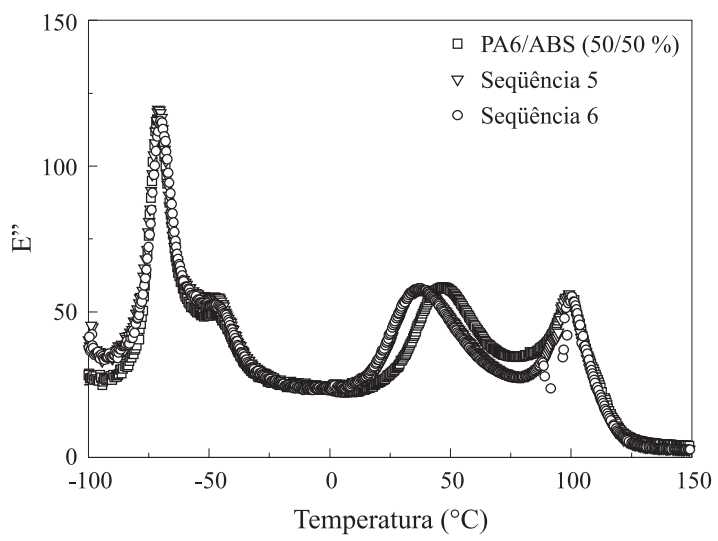

Figura 6. Comportamento do módulo de perda em função da temperatura para as blendas PA6/ABS/MMA-GMA (47,5/47,5/5\%).

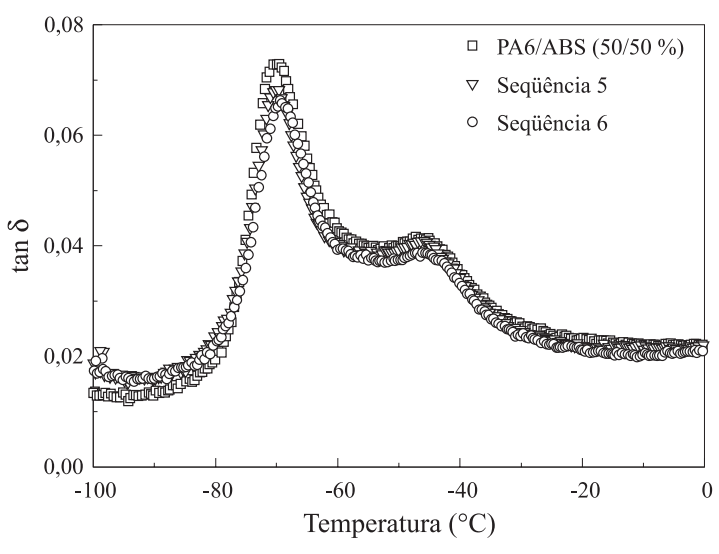

(a)

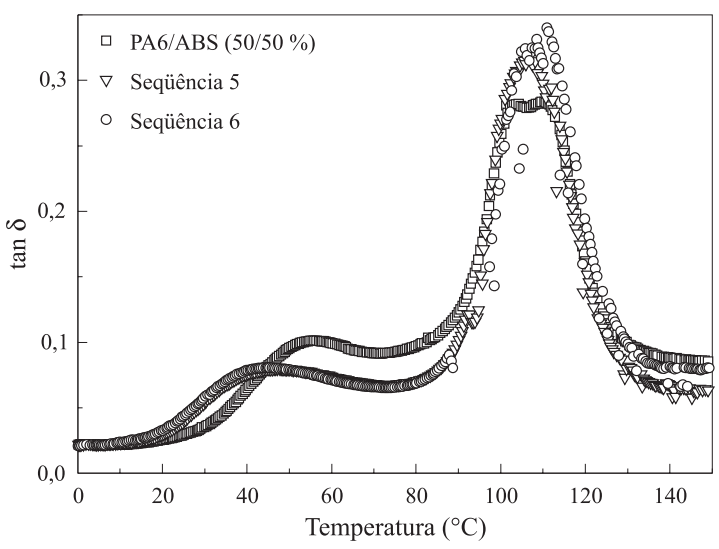

(b)

Figura 7. Curvas de $\tan \delta$ em função da temperatura para as blendas PA6/ ABS/MMA-GMA (47,5/47,5/5\%). Em (a) intervalo de temperatura de 100 a $0{ }^{\circ} \mathrm{C}$; em (b) intervalo de temperatura de 0 a $150{ }^{\circ} \mathrm{C}$.

dentro do erro experimental. As blendas compatibilizadas com o copolímero MMA-GMA, seqüências 1, 2, 3 e 4 apresentam uma redução significativa na $\mathrm{Tg}$ em relação ao SAN. Todas as seqüências estudadas apresentam uma redução significativa na Tg em relação à PA6 pura e à PA6 nas blendas compatibilizadas com o copolímero MMA-MA. Isso pode estar relacionado aos vários fatores já mencionados como: o caráter difuncional da PA6; a polimerização por abertura de anel que pode ocorrer com os grupos epóxis na presença de um iniciador; a presença de emulsificante no ABS que pode favorecer as reações com epóxis ${ }^{[19]}$. Todos esses fatores podem ter degenerado o sistema, reduzindo a massa molar e alterando de forma significativa a $\mathrm{Tg}$, já que esta é função da estrutura molecular do polímero. Isso também pode ser confirmado pelos resultados de $\mathrm{DSC}^{[1]}$ através da redução no grau de cristalinidade da PA6 com a presença do copolímero MMA-GMA, ou seja, este copolímero pode modificar as características das moléculas de PA6 sob cristalização. A presença do copolímero MMAMA na blenda, entretanto, não alterou de forma significativa as Tg's da fase SAN. Como pode ser observado na Tabela, o deslocamento da Tg da PA6 para temperaturas maiores, comparado às blendas com o copolímero MMA-GMA, pode estar relacionado ao aumento no conteúdo de cristalinidade desta 
fase. Os resultados de DSC revelam que o copolímero MMAMA parece contribuir, de alguma forma, para o processo de cristalização da PA ${ }^{[1,36]}$. Esses dados podem evidenciar que a compatibilidade das duas fases, PA6 e ABS, é melhorada pela presença do MMA-MA ${ }^{[37]}$.

\section{Conclusões}

O objetivo deste trabalho foi produzir blendas tenazes PA6/ ABS, de grande interesse comercial, utilizando-se um novo sistema de compatibilização à base de copolímeros acrílicos (MMA-GMA e MMA-MA) capazes de formar um copolímero enxertado de PA6-g-copolímero na interface, rica entre as fases de PA6 e ABS. O copolímero enxertado funcionaria como uma ponte impedindo a coalescência dos domínios de ABS, diminuindo estes, estabilizando a morfologia e tenacificando o sistema como um todo. Entretanto, foi observado que o copolímero à base de GMA, não foi tão efetivo para o sistema específico. A blenda com esse copolímero, apresentou-se dúctil na temperatura ambiente, mas frágil em temperaturas logo abaixo desta. O grupo epóxi presente no GMA é capaz de reagir com os grupos terminais amina da PA6 e também com os grupos terminais ácidos. Este talvez tenha sido o grande problema encontrado para a compatibilização efetiva do sistema que como observado pela análise morfológica, os domínios de $\mathrm{ABS}$ não diminuíram o suficiente para a tenacificação da blenda. Conseqüentemente, as propriedades mecânicas não foram as desejadas. As blendas com o copolímero à base de MA, entretanto, possui o grupo anidrido capaz de reagir apenas com os grupos terminais amina da PA. Foi observada, pela análise morfológica, uma estrutura constituída de uma fase bem dispersa e distribuída na matriz de PA6, significativamente diferente daquela obtida para a blenda sem compatibilizante. As propriedades mecânicas destas blendas foram excelentes, comparáveis às obtidas na literatura. Tais resultados são evidência de que o copolímero MMA-MA, até então não utilizado para este tipo de blendas, constitui uma alternativa eficiente de compatibilização reativa para o sistema PA6/ABS, por ser miscível com a matriz SAN do ABS e ser capaz de formar reação com os grupos terminais amina da PA6.

\section{Agradecimentos}

Os autores agradecem à Basf pelo fornecimento da poliamida 6, à Bayer pelo fornecimento do ABS e à Nitriflex pelo uso de seus laboratórios para a caracterização do mesmo, ao PRONEX (Núcleo de Reologia e Processamento de Polímeros - DEMa/ UFSCar) e à FAPESP pelo apoio financeiro.

\section{Referências Bibliográficas}

1. Araújo, E.M. - “Tenacificação da poliamida 6 com ABS por meio da técnica de compatibilização in situ com o uso de copolímeros acrílicos reativos", Tese de Doutorado, Universidade Federal de São Carlos, Brasil (2001).
2. Triacca, V.J.; Ziaee, S.; Barlow, J.W.; Keskkula, H. \& Paul, D.R - Polymer, 32, p.1401 (1991).

3. Hage Jr., E.; Hale, W.; Keskkula, H. \& Paul, D.R. Polymer, 38, p.3237 (1997).

4. Jang, S. P. \& Kim, D. - Polym. Engng. Sci., 40, p.1635 (2000).

5. Misra, A.; Sawhney, G. \& Kumar, R.A. - J. Ap. Pol. Sci., 50, p.1179 (1993).

6. Majumdar, B.; Keskkula, H. \& Paul, D. R. - Polym. Prepr., 35, p.850 (1994).

7. Majumdar, B.; Keskkula, H. \& Paul, D.R. - J. Pol. Sci.: part B: pol. phys., 32, p.2127 (1994).

8. Majumdar, B.; Keskkula, H. \& Paul, D.R. - Polymer, 35, p.5453 (1994).

9. Majumdar, B.; Keskkula, H. \& Paul, D.R. - Polymer, 35, p.5468 (1994).

10. Majumdar, B.; Keskkula, H. \& Paul, D.R. - J. App. Pol. Sci., 54, p.339 (1994).

11. Araújo, E.M.; Hage Jr., E. \& Carvalho, A.J.F. - Polímeros Ciência e Tecnologia, 13, n. 3, p.205 (2003).

12. Carrot, C.; Guillet, J. \& May, J.F. - Plast. Rubber Comp. Proc. Appl., 16, p.61 (1991).

13. Kim, B.K.; Lee, Y.M. \& Jeong, H.M. - Polymer, 34, p.2075 (1993).

14. Gan, P.P. \& Paul, D.R. - Polymer, 35, p. 3513 (1994).

15. Gan, P.P.; Paul, D.R. \& Padwa, A.R.- Polymer, 35, p. 1487 (1994).

16. Kim, J.K. \& Lee, H. - Polymer, 37, p. 305 (1996).

17. Kudva, R.A.; Keskkula, H. \& Paul, D.R. - Polymer, 39, p.2447 (1998).

18. Lee, P.-C.; Kuo, W.-F. \& Chang, F.-C. - Polymer, 35, p. 5641 (1994).

19. Hale, W.; Keskkula, H. \& Paul, D.R. - Polymer, 40, p. 365 (1999).

20. Maa, C.-T. \& Chang, F.-C. - J. Ap. Pol. Sci., 49, p. 913 (1993).

21. Lu, M.; Keskkula, H. \& Paul, D.R. - Polym. Engng. Sci., 34, p. 33 (1994).

22. Liu, W.B. \& Chang, F.C. - Polymer Preprints, 34, p. 803 (1993).

23. Chen, S.-H. \& Chang, F.-C. - J. Ap. Pol. Sci., 51, p. 955 (1994).

24. Bassani, A. - "Tenacificação de polipropileno através da incorporação de copolímero em bloco de estireno/etileno -butileno/estireno (SEBS) e de SEBS funcionalizado 
com anidrido maléico (SEBS-g-MA)", Dissertação de Mestrado, Universidade Federal de São Carlos, Brasil (1998).

25. Okada, O.; Keskkula, H. \& Paul D.R. - Polymer, 41, p. 8061 (2000).

26. Oshinski, A.J.; Keskkula, H. \& Paul, D.R. - Polymer, 37, p. 4909 (1996).

27. Kudva, R.A.; Keskkula, H. \& Paul, D.R. - Polymer, 40, p.6003 (1999).

28. Oshinski, A.J.; Keskkula, H. \& Paul, D.R. - Polymer, 37, p. 4919 (1996).

29. Oswald, T.A. \& Menges, G. - "Materials Science of Polymers for Engineers", Hanser Publishers, New York (1996).

30. Bucknall, C.B. - “Toughened Plastics”, Applied Science Publ., London (1977).

31. Paul, D.R. \& Newman, S. - "Polymer Blends", Academic Press, New York (1978).
32. Ferreira, L.A.S. - “Comportamento térmico, mecânico e termo-mecânico de blendas poliméricas constituídas de polibutileno tereftalato (PBT) e copolímero acrilontrilabutadieno-estireno (ABS)", Dissertação de Mestrado, Universidade Federal de São Carlos, Brasil (1995).

33. Canevarolo, S.V.- Análise dinâmico-mecânica para sistemas poliméricos. Parte 1 - Amortecimento (tan ઈ) Boletim, 6, ABPol, p. 37 (1991).

34. Khanna, Y.P. - Macromolecules, 25, p. 3598 (1992).

35. Bonse, B.C. - “Caracterização de estado de miscibilidade de blendas poliméricas constituídas de policarbonatos e copolímero SAN", Dissertação de Mestrado, Universidade Federal de São Carlos, Brasil (1999).

36. Araújo, E.M.; Hage Jr., E. \& Carvalho, A.J.F. - J. Mat. Sci., v.39, p. 1173 (2004).

37. Huang, Y.; Liu, Y. \& Zhao, C. - J. App. Pol. Sci., 69, p. 1505 (1998).

Recebido: 01/08/03

Aprovado: 20/11/03 